\title{
Performance Evaluation of Digital Modulation Techniques on DS-WCDMA
}

\author{
Khalid Hamid \\ University of science and \\ Technology
}

\author{
Ibrahim Khider \\ Sudan University of Science \\ and Technology
}

\author{
Amin Babiker \\ Elneilein University
}

\begin{abstract}
The objectives of this paper are to study, analyze and evaluate the performance of Direct Sequence - Wide Code Division Multiple Access (DS-WCDMA) systems in Mobile Rayleigh fading propagation channel. The Parameters which are considered for the evaluation performance are Additive white Gaussian noise (AWGN), Rayleigh propagation fading channel and the Code sequence .length. The evaluation of DSWCDMA was derived for different type of digital modulation using Mat lab simulation link. The results were obtained in terms of bit error rates(BER)in graphical form.
\end{abstract}

\section{Keywords}

DS; WCDMA; AWGN; SIMULATION; QAM ;QPSK

SNR BER BPSK

\section{INTRODUCTION}

W-CDMA uses noise-like broadband frequency spectrum where it has high resistance to multipath fading where as this was not present in conventional narrowband signal of 2 nd generation (2G) communication system[1]. However, the implementation of high data rate modulation techniques that have good bandwidth efficiency in W-CDMA cellular communication requires perfect modulators, demodulators, filter and transmission path that are difficult to achieve in practical radio environment. Modulation schemes which are capable of delivering more bits per symbol are more immune to errors caused by noise and interference in the channel. Moreover, errors can be easily produced as the number of users is increased and the mobile terminal is subjected to mobility. Thus, it has driven many researches into the application of higher order modulations [2] [3].With growing popularity and usage of the third generation networks, the cost efficient optimization of network capacity and quality of service will become essential to cellular operators. This is achieved with careful network planning and operation, improvements in transmission methods, and advances in receiver techniques. [4]WCDMA is a promising technique for achieving the high data capacity and spectral efficiency requirements for wireless communication system of the near future. [5]W-CDMA is being used by Universal Mobile Telecommunication System (UMTS) as platform of the 3rd generation cellular communication system. High data rate signal transmission can be transmitted over the air by using W-CDMA system, thus enabling of multimedia rich applications such as video streams and high resolution pictures to end users. Thus, we need suitable modulation technique and error correction mechanism to be used in $\mathrm{W}$ CDMA system.[6]WCDMA is the most commonly adopted radio interface in third generation Universal Mobile Telecommunications System (UMTS) networks. UMTS and WCDMA are widely described in [8] and in 3GPP specifications [7]. Uplink and downlink packet evolutions, HSUPA and HSDPA respectively, to WCDMA are introduced in detail in [8], [9] and [10]. W-CDMA systems can employ the high order modulation (MPSK or M-QAM) to increase the transmission data rate with the link quality control.[6] .

\section{System Model}

The system model is depicted in Fig 1.

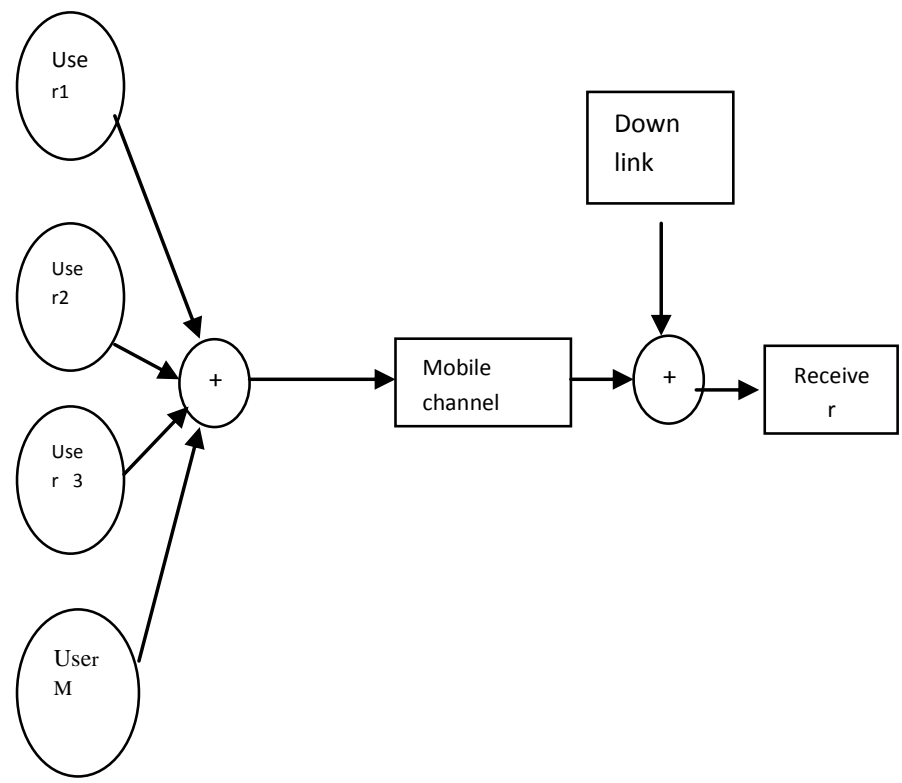

Fig (1): DS- WCDMA system model 
In DS-WCDMA, users employ their own sequence to spread the information data. The information data of each user is modulated by narrowband digital modulation then the first bits of modulated data is spreaded by the code sequence and the spreaded data of all users are transmitted to the base station at the same time. The base station detects the information data of each user by correlating the received signal with the code sequence allocated to each user. The transmitted data in the in-phase channel and Quadrature phase modulated by digital modulation are multiplied by the code sequence used to spread the transmitted data then the transmitted signal is contaminated in a Rayleigh fading channel.

At the receiver, AWGN is added to the received data, the resample data are the data of all users. By correlating the data with the spread code used at the transmitter, the transmitted data of all users is detected.

\section{Mathematical Model}

Provide an expression to determine the average bit error rate for user in a single channel, WCDMA mobile radio system.

In WCDMA system using binary signalling the radio signal received at the base station from the kth mobile user is given by:[11]

$S_{K}\left(t-\tau_{k}\right)=\sqrt{2 P_{k}} a_{k}\left(t-\tau_{k}\right) b_{k}\left(t-\tau_{k}\right) \cos \left(\omega_{c} t+\phi_{k}\right)$

Where:

$b_{k}$ :

data sequence for user $\mathrm{k}=[-1,1]$

$\mathrm{a}_{\mathrm{k}}$ : spreading sequence for user $\mathrm{k}=[-1,1]$

$\tau_{\mathrm{k}}$ :is delay for user $\mathrm{k}$ relative to some reference for user 0

$\mathrm{P}_{\mathrm{k}}$ :is the received power of user $\mathrm{k}$

$\Phi_{\mathrm{k}}$ :is the carrier phase offset for user $\mathrm{k}$ relative to reference user 0

At the receiver the signal available to the input to the correlator is given by:

$r(t)=\sum S_{k}\left(t-\tau_{k}\right)+n(t)$

Where $n(t)$ is AWGN with spectral density $\mathrm{N}_{0} / 2$

The received signal contains both the desired user and $(\mathrm{k}$ 1) undesired users and is mixed down to base band multiplied by $\mathrm{PN}$ sequence of the desired user and integrated over one period.

Assuming that the receiver is delayed and phase synchronized user 0 the decision static user 0 is given by:

$$
Z_{0}=\int_{j T_{b}}^{(j+1) T_{b}} r(t) a_{0}(t) \cos \omega_{c} d t
$$

May be expressed as :

$Z=I_{0}+\eta+\zeta$

where $I_{0}$ :is desired contribution of to decision statistic from user $\mathrm{k}=0$

$$
\left.I_{0}=\sqrt{2 p_{0}} \int_{t=0}^{T_{b}} a_{k}^{2}(t) b_{k}(t) \cos ^{2} \omega_{c} t d t=\frac{\sqrt{P_{0}}}{2} b_{k, 0} T_{b} \ldots \ldots \ldots \ldots \ldots \ldots \ldots \ldots \ldots\right)
$$

$\xi_{\text {:is multiple access interference from all cochannel }}$ users

$\eta:$ is thermal noise contribution(AWGN)

$$
\eta=\int_{t=0}^{T_{b}} n(t) a_{0}(t) \cos \omega_{c} t
$$

With mean $\mu_{\eta=0}$ and variance $=\sigma_{\eta}=\frac{N_{0} T_{b}}{4}$ for $\omega_{c}>>\frac{2}{T_{b}}$

The multiple access interference is the summation of $\mathrm{K}-1$ termsIk $\xi=\sum_{k=1}^{k-1} I_{k}$

each of which is given by:

$\left.I_{k}=\int_{t=0}^{T_{b}} \sqrt{2 P_{k}} a_{k}\left(t-\tau_{k}\right) b_{k}\left(t-\tau_{k}\right) \cos \left(\omega_{c} t+\phi_{k}\right)+n(t)\right] a_{0}(t) \cos \left(\omega_{c} t+\phi_{k}\right) \cos \omega_{c} t d t$. .$(8)$

The average bit error rate probability is given by:

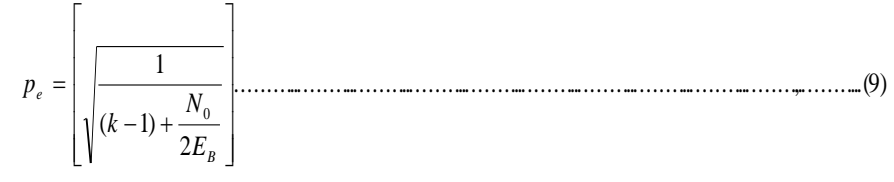

\section{Simulation}

A computer program using Matlab software program is implemented to simulate the BER performance in a AWGN and Rayleigh fading environments of DS-WCDMA.

The DS- WCDMA model is simulated for s gold spreading sequences code in the presence of Rayleigh channel by considering multi users and binary, Quadrate Phase Shift Keying (BPSK \&QPSK) and M-array-QAM as modulation scheme. The simulation parameters used in the simulation are given in Table 1. Then the BER performance of DS-WCDMA is calculated and compared by varying the length of above mentioned sequences. The BER is the number of bit received as errors divided by the total number of transmitted bits during a studied time interval

TABLE 1 ,Simulation environment

\begin{tabular}{|l|l|}
\hline \multicolumn{1}{|c|}{ Parameter } & \multicolumn{1}{c|}{ Value } \\
\hline Spreading code & M-Sequence \\
\hline Code length & $63,127,255,511$ \\
\hline NO. of users & FROM 1 TO 100 \\
\hline Modulation techniques & BPSK,QPSK,16-QAM \\
\hline SNRdB & FROM 0 TO 40 dB \\
\hline Channel & Rayleigh channel with AWGN \\
& \\
\hline
\end{tabular}




\section{RESULT AND DISCUSSION}

Fig (1): below shows the performance results for single user WCDMA and QPSK, BPSK,16QAM digital modulation in mobile AWGN and Rayleigh fading ,the result shows the bit error rate decrease as signal to noise increased. And fig(2):shows that the bit error rate decrease as the code length increase on the performance of WCDMA/BPSK while fig(3): shows that the bit error rate increased as the number of users increased for WCDMA with different type digital modulation.

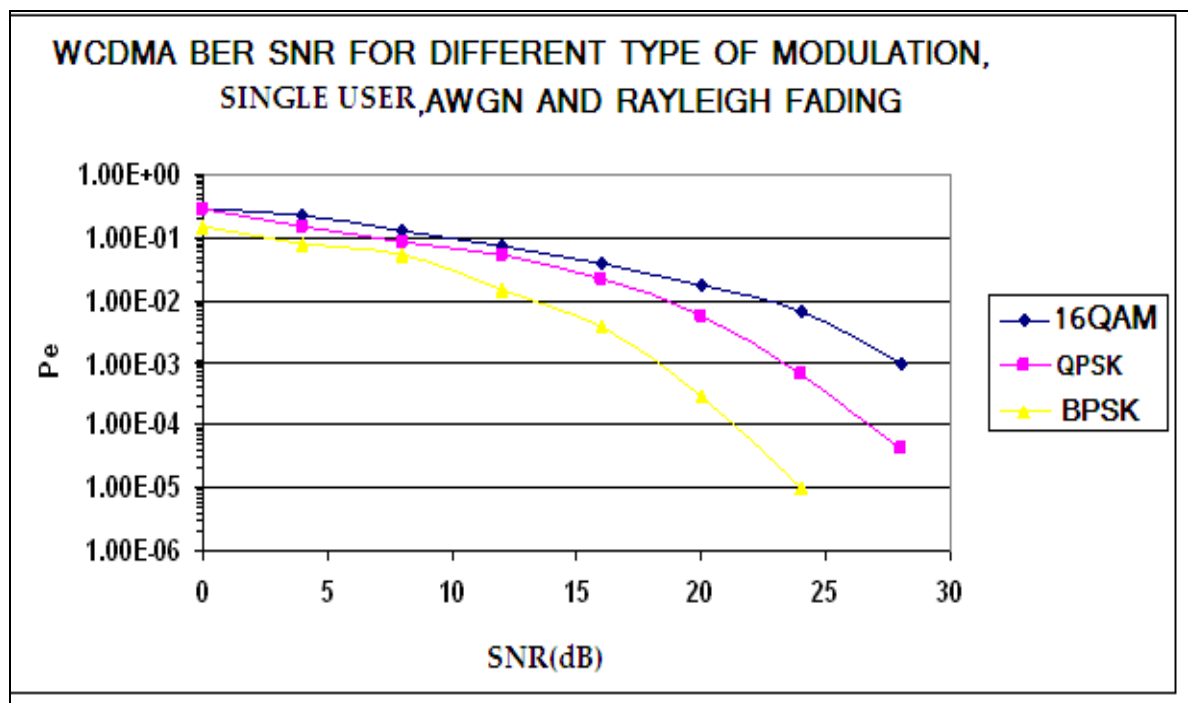

Fig(2): performance of WCDMA against Signal to noise ratio for AWGN and Rayleigh fading

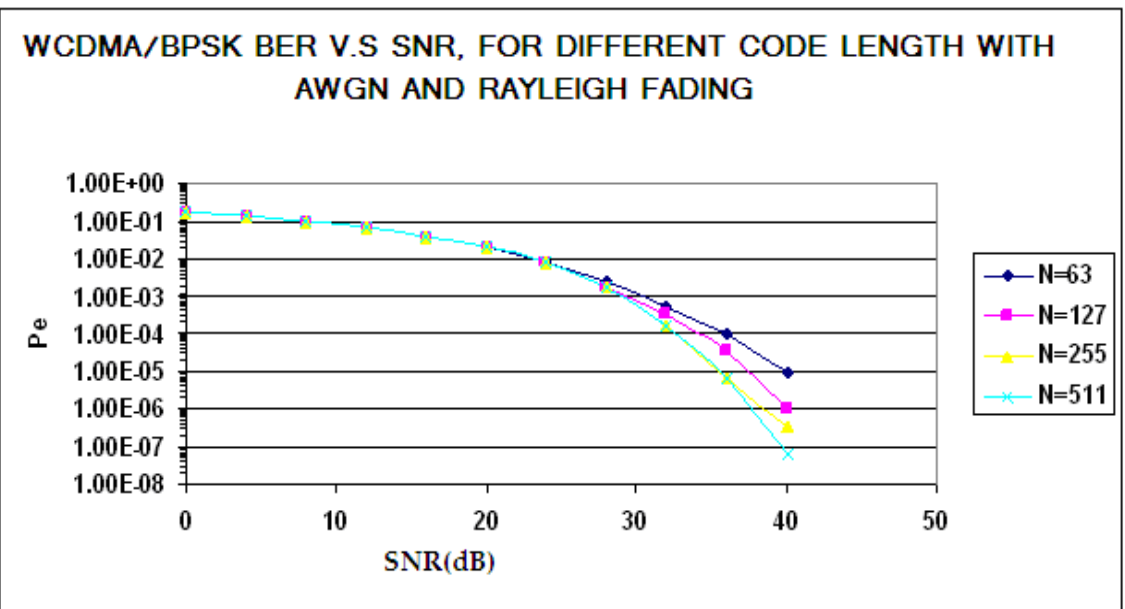

Fig(3):performance of WCDMA/BPSK with different code length, AWGN AND Rayleigh fading 


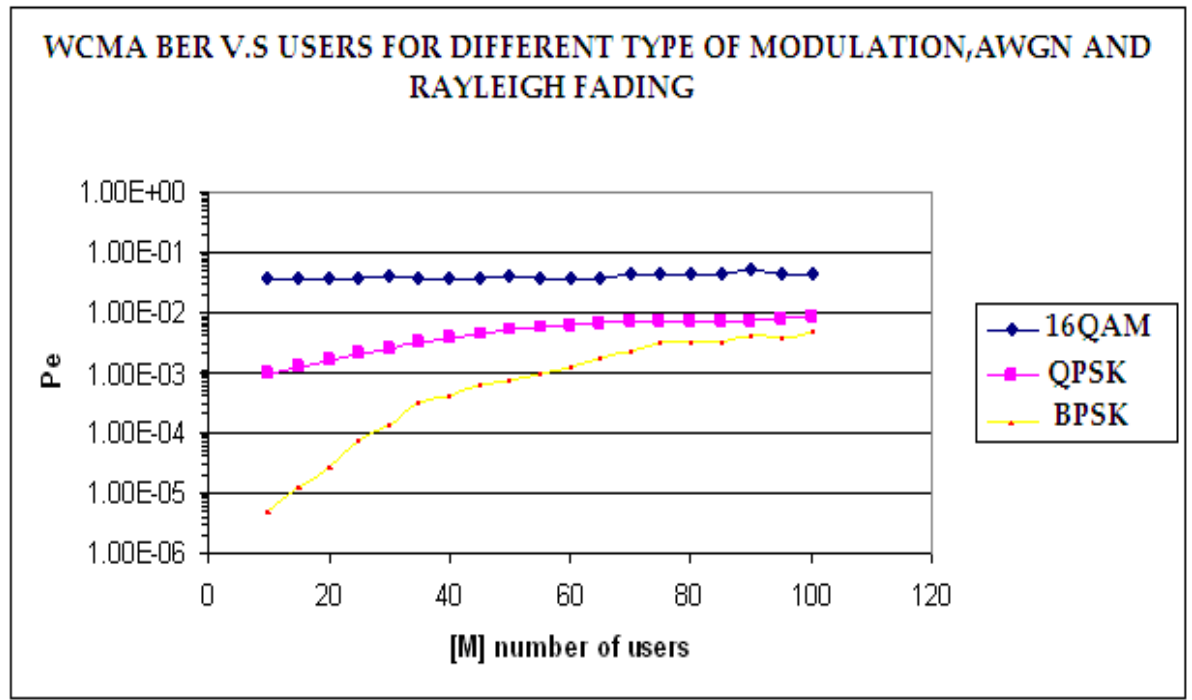

Fig(4): performance of WCDMA against Number of users with AWGN and Rayleigh fading channel

\section{CONCLUSION}

In this paper we analyze three modulation techniques,BPSK, QPSK and 16-QAM to reduce the error performance of the signal and compare which technique is better through Rayleigh Fading Channel in the presence of AWGN.The performance of W-CDMA system in AWGN channel shows that BPSK modulation technique has a better performance compared to that of QPSK and 16-QAM. The performance of BPSK,QPSK and 16-QAM modulation technique in WCDMA system degrades. As the However, QPSK shows better performance compared to that of 16-QAM in LOS channel and multipath Rayleigh fading channel. In other words, 16QAM suffers signal degradation and error proned when the simulations are done in these channels. As the number of users is increased, the BPSK modulation technique performs poorly in W-CDMA system. It is expected that 16-QAM will show performance degradation similar like QPSK as the number of users is increased but with lower performance compared to that of QPSK. In general, the reason that causes poor performance of W-CDMA system when the number of users increased is because the value of cross correlation between the codes is not 0 and thus it causes interference. Many studies and researches have showed that 16-QAM modulation technique is a primary candidate for high speed data transmission in $3 \mathrm{G}$ mobile communication. However, higher data rate modulation scheme (e.g.16-QAM) suffers significant degradation in noise and Multipath Rayleigh fading channel compared to lower data rate modulation technique (e.g. QPSK). The errors are resulted from interference between adjacent carriers phase in constellation of M-ary QAM. Larger value of M of M-ary QAM suffers more signal degradation. Thus, it is suggested that high data rate modulation technique such as 16-QAM needs an error correction coding such as convolutional coding or turbo coding so that the interference from the adjacent carrier phase in the constellation of 16-QAM can be eliminated if not minimized.

\section{REFERENCES}

[1] M. A. Masud, M. Samsuzzaman, M. A.Rahman,2010, "bit Error Rate Performance Analysis onModulation Techniques of Wideband CodeDivision Multiple Access"”,", JOURNAL OF TELECOMMUNICATIONS,

[2] Y.Rosmansyah, P. Sweeney, R. Tafazolli,2001,“AirInterface Techniques for Achieving High Data Rates for UMTS", IEEE $3 \mathrm{G}$ Mobile Communication Technologies.

[3] E. Hossain, T. Issariyakul,2004,"Performance bound of dynamic forward link adaptation in cellular W-CDMA networks using high-order modulation and multicode formats", IEEE Electronics Letters.

[4] Hooli, Kari,2003, Equalization in WCDMA Terminals Department of Electrical and Information Engineering, University of Oulu, P.O.Box 4500, FIN-90014 University of Oulu, Finland.

[5] Dr. M. Mahbubur Rahman, Md. Khairul Islam, Tarek Hassan-Al-Mahmud, A. R. Mahmud,2012, ,PERFORMANCE ANALYSIS OF DOWNLINK POWER CONTROL IN WCDMA SYSTEM International Journal of Scientific \& Engineering Research Volume 3, Issue 8.

[6] Aun Ali Tahir,Feng Zhao,2009, PERFORMANCE ANALYSIS ON MODULATION TECHNIQUES OF W-CDMA IN MULTIPATH FADING CHANNEL, thesis of MSc in Electrical Engineering Blekinge Institute of Technology.

[7] http://www.3gpp.org/, Cited June 2008

[8] H. Holma and A. Toskala,2007, WCDMA for UMTS: Radio Access for Third Generation Mobile Communications, 4th edition. John Wiley \& Sons Ltd, 2007.

[9] H. Holma and A. Toskala, HSDPA/HSUPA for UMTS, 1st edition, John Wiley \& Sons Ltd, 2006.

[10] E. Dahlman, S. Parkvall, J. Sköld and P. Beming, 3G Evolution - HSPA and LTE for Mobile Broadband, 1st edition, Elsevier Ltd..

[11] Theodore S.Rappaport,2002,"Wireless communications prinviple and practice,prentice Hall. 